\title{
Analyzing power for the proton elastic scattering from neutron-rich ${ }^{6} \mathrm{He}$ nucleus
}

\author{
T. Uesaka, ${ }^{1, *}$ S. Sakaguchi, ${ }^{1}$ Y. Iseri, ${ }^{2}$ K. Amos,${ }^{3}$ N. Aoi,${ }^{4}$ Y. Hashimoto,${ }^{5}$ E. Hiyama, ${ }^{4}$ M. Ichikawa,${ }^{6}$ \\ Y. Ichikawa, ${ }^{7}$ S. Ishikawa, ${ }^{8}$ K. Itoh, ${ }^{9}$ M. Itoh, ${ }^{6}$ H. Iwasaki, ${ }^{7}$ S. Karataglidis,${ }^{10}$ T. Kawabata,${ }^{1}$ \\ T. Kawahara, ${ }^{11}$ H. Kuboki,${ }^{7}$ Y. Maeda,${ }^{12}$ R. Matsuo,${ }^{6}$ T. Nakao, ${ }^{7}$ H. Okamura, ${ }^{13}$ H. Sakai, ${ }^{7}$ \\ Y. Sasamoto, ${ }^{1}$ M. Sasano, ${ }^{7}$ Y. Satou, ${ }^{5}$ K. Sekiguchi, ${ }^{4}$ M. Shinohara, ${ }^{5}$ K. Suda, ${ }^{13}$ D. Suzuki, ${ }^{7}$ \\ Y. Takahashi, ${ }^{7}$ M. Tanifuji, ${ }^{8}$ A. Tamii, ${ }^{13}$ T. Wakui, ${ }^{6}$ K. Yako, ${ }^{7}$ Y. Yamamoto,,${ }^{14}$ and M. Yamaguchi ${ }^{4}$ \\ ${ }^{1}$ Center for Nuclear Study, University of Tokyo, Tokyo 113-0033, Japan \\ ${ }^{2}$ Department of Physics, Chiba-Keizai College, Chiba 263-0021, Japan \\ ${ }^{3}$ School of Physics, University of Melbourne, Melbourne, Australia \\ ${ }^{4}$ RIKEN Nishina Center, Saitama 351-0198, Japan \\ ${ }^{5}$ Department of Physics, Tokyo Institute of Technology, Tokyo 152-8551, Japan \\ ${ }^{6}$ Cyclotron 85 Radioisotope Center, Tohoku University, Miyagi 980-8578, Japan \\ ${ }^{7}$ Department of Physics, University of Tokyo, Tokyo 113-0033, Japan \\ ${ }^{8}$ Science Research Center, Hosei University, Tokyo 102-8160, Japan \\ ${ }^{9}$ Department of Physics, Saitama University, Saitama 338-8570, Japan \\ ${ }^{10}$ Department of Physics and Electronics, Rhodes University, P.O. Box 94 Grahamstown 6140, South Africa \\ ${ }^{11}$ Department of Physics, Toho University, Chiba 274-8510, Japan \\ ${ }^{12}$ Faculty of Engineering, University of Miyazaki, Miyazaki 889-2192, Japan \\ ${ }^{13}$ Research Center for Nuclear Physics, Osaka University, Osaka 567-0047, Japan \\ ${ }^{14}$ Tsuru University, Yamanashi 402-8555, Japan
}

(Dated: October 22, 2018)

\begin{abstract}
Vector analyzing power for the proton- ${ }^{6} \mathrm{He}$ elastic scattering at $71 \mathrm{MeV} /$ nucleon has been measured for the first time, with a newly developed polarized proton solid target working at low magnetic field of $0.09 \mathrm{~T}$. The results are found to be incompatible with a $t$-matrix folding model prediction. Comparisons of the data with $g$-matrix folding analyses clearly show that the vector analyzing power is sensitive to the nuclear structure model used in the reaction analysis. The $\alpha$-core distribution in ${ }^{6} \mathrm{He}$ is suggested to be a possible key to understand the nuclear structure sensitivity.
\end{abstract}

PACS numbers: 24.70.+s, 25.60.-t, 29.25.Pj

Spin observables in scattering experiments have been rich sources of our understanding of nuclear structure, reaction, and interactions. One of the good examples is spin asymmetry in proton-proton and proton-nucleus $(p-A)$ scatterings which is a direct manifestation of spinorbit coupling in the system. The first spin asymmetry measurements carried out by use of a double scattering method [1, 2] clearly demonstrated that the spin-orbit coupling in nuclei is an order of magnitude stronger than that due to the relativistic effect [3]. At present the spin-orbit coupling in $p$ - $A$ scattering is quantitatively established through numerous experiments using polarized proton beams for stable targets.

It is interesting to use spin asymmetry measurements to study unstable nuclei. Nuclei locating near the neutron drip line occasionally show distinctive structure such as halos or skins. The neutron rich ${ }^{6} \mathrm{He}$ nucleus is one of the typical nuclides with an extended neutron distribution. Since the extended neutron distribution is prominent at the nuclear surface and the spin-orbit coupling is, in nature, a surface phenomenon, it is stimulating to see how the extended neutron distributions affect the spin asymmetry, i.e., vector analyzing power in proton elastic scattering.

In this Letter, we report new results of vector analyzing power for the $p_{-}{ }^{6} \mathrm{He}$ elastic scattering at $71 \mathrm{MeV} /$ nucleon, measured with a newly developed polarized proton target. The results are compared with microscopic folding model calculations.

Although cross sections in proton elastic scattering from ${ }^{6} \mathrm{He}$ have been extensively measured over a wide range of energies [4 9], until recently there had been no measurement of vector analyzing power. Since unstable nuclei are produced as secondary beams, we need a polarized proton target, practically in the solid state, for the spin-asymmetry studies. In addition, the solid polarized proton target should work under a low magnetic field of $B \sim 0.1 \mathrm{~T}$ for detection of recoiled protons with magnetic rigidity as low as $0.3 \mathrm{Tm}$. The traditional dynamical nuclear polarization technique [10], demanding a magnetic field higher than a few Tesla, can not be applied therefore. Although this difficulty might be overcome by applying a "spin frozen" operation, efforts to do so have not been successful so far. An alternative approach to overcome the problem is to develop a polarized target based on a new principle which is independent of magnetic field strength.

We have succeeded in constructing a new solid polarized proton target working at a low magnetic field of about $0.1 \mathrm{~T}$ [11]. Here, protons in the target are polarized by transferring electron polarization in photoexcited triplet states of pentacene molecules via cross 
polarization 12]. The magnitude of the electron polarization is $73 \%$ and depends neither on the magnetic field strength nor on the temperature of material. This makes it possible to operate the polarized target under a low magnetic field of $0.1 \mathrm{~T}$ and a high temperature of $100 \mathrm{~K}$.

The first experiment with this target system was carried out in 2003 where spin asymmetry in the $p-{ }^{6} \mathrm{He}$ elastic scattering was measured[15]. The data presented interesting features which were completely incompatible with theoretical predictions. From phenomenological optical model analyses, it was implied that the $p-{ }^{6} \mathrm{He}$ spinorbit potential might extend to a larger radius compared with the $p-{ }^{6} \mathrm{Li}$ case. In Ref. [16], Crespo and Moro claim that an extended neutron distribution cannot be responsible for the large spin-orbit radius. Thus connection between spin-orbit potential in the $p^{-}{ }^{6} \mathrm{He}$ scattering and the extended neutron distribution in ${ }^{6} \mathrm{He}$ is still unclear. However, accuracy of the previous data is insufficient for further detailed and quantitative analysis. This is mainly because the analyzing power data were obtained with an assumed value of target polarization [15].

To obtain accurate analyzing power data with a reliable normalization, we have performed the $p-{ }^{6} \mathrm{He}$ spinasymmetry experiment with an upgraded target and detector systems.

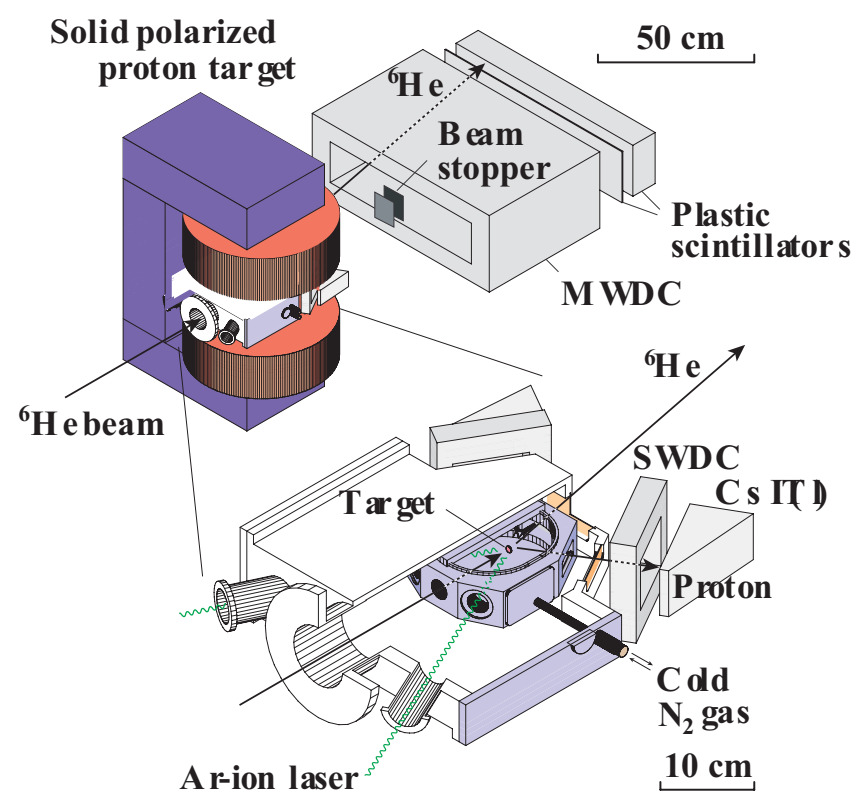

FIG. 1: (color online) Schematic layout of the experimental setup, including the polarized proton solid target.

The experiment was performed at RIKEN Accelerator Research Facility. The setup including the polarized target system, shown in Fig. 1 was placed downstream of the final focal plane of RIPS [13].

A radioactive ${ }^{6} \mathrm{He}$ beam with an energy of $70.6 \pm 1.4 \mathrm{MeV} /$ nucleon was produced via the projectile fragmentation reaction of a primary ${ }^{12} \mathrm{C}$ beam on a 1.39 $\mathrm{g} / \mathrm{cm}^{2}$ beryllium target. An energy and an average intensity of the primary beam were $92 \mathrm{MeV} /$ nucleon and $600 \mathrm{pnA}$, respectively. The resulting intensity and purity of ${ }^{6} \mathrm{He}$ beam was $3.0 \times 10^{5}$ cps and $95 \%$, respectively.

After separation in RIPS, the ${ }^{6} \mathrm{He}$ beam bombarded the polarized target made of a crystal of naphthalene with a small amount $(\sim 0.005 \mathrm{~mol} \%)$ of pentacene as a dopant. The dimensions of the target were $1 \mathrm{~mm}$ in thickness and $14 \mathrm{~mm}$ in diameter. The target was placed in a homogeneous magnetic field of $0.09 \mathrm{~T}$ produced by a C-type magnet. The target chamber which was thermally isolated from the room-temperature environment and was cooled down to $100 \mathrm{~K}$ by blowing a cold nitrogen gas. Laser light from Ar-ion lasers irradiated the target to polarize electrons in pentacene molecules [14].

The relative magnitude of the proton polarization was monitored with a pulse NMR method during the measurement. The absolute value of the polarization was calibrated by comparing the NMR signal amplitude to the asymmetry of the $p-{ }^{4} \mathrm{He}$ scattering, measured with the same setup at $80 \mathrm{MeV} /$ nucleon. The analyzing power data for the $p-{ }^{4} \mathrm{He}$ scattering in Ref. [17] were used in the calibration. The proton polarization was found to be $20 \pm 4 \%$ at maximum and $14 \pm 3 \%$ on average. Statistical uncertainty in the $p-{ }^{4} \mathrm{He}$ measurement dominates the uncertainties in the proton polarization.

Scattered ${ }^{6} \mathrm{He}$ particles were detected by a multi-wire drift chamber (MWDC) and plastic scintillators placed about $1 \mathrm{~m}$ downstream of the target. Pulse height information from the plastic scintillators is used to identify the particle. The ${ }^{6} \mathrm{He}$ trajectory determined by MWDC provides the scattering angle of the ${ }^{6} \mathrm{He}$ and the reaction position on the target. Two counter telescopes to detect recoiled protons were placed left and right with respect to the beam axis. Each telescope consisted of a singlewire drift chamber (SWDC) for a position measurement and a $\mathrm{CsI}(\mathrm{Tl})$ scintillator for a total energy measurement. They covered an angular range of $\theta_{\text {c.m. }}=35^{\circ}-90^{\circ}$ in the center of mass system. The background around the elastic scattering peak was reasonably small, which enables us to obtain yields of interest reliably.

The angular distributions of differential cross section $(d \sigma / d \Omega)$ and vector analyzing power $\left(A_{y}\right)$ are shown by filled circles in Fig. 2. Only statistical uncertainties are shown in the figure. Systematic uncertainty in $A_{y}$, mainly due to uncertainty in absolute normalization of proton polarization, is $19 \%$ independent of scattering angles. The cross section data are obtained with systematic uncertainty of $9 \%$ up to backward angles of $\theta_{\text {c.m. }} \leq 87^{\circ}$.

In the top panel of the figure, the data for the $p{ }^{6} \mathrm{Li}$ elastic scattering [19] (triangles) are shown for comparison. As is indicated in Ref.[15], $d \sigma / d \Omega$ in the $p-{ }^{6} \mathrm{He}$ scattering is almost identical to that in the $p$ - ${ }^{6} \mathrm{Li}$ scattering at $\theta_{\text {c.m. }} \leq 50^{\circ}$. This indicates that matter distributions in ${ }^{6} \mathrm{He}$ and ${ }^{6} \mathrm{Li}$ are similar, which is consistent with the recent results from GSI [8]. On the other hand, one 


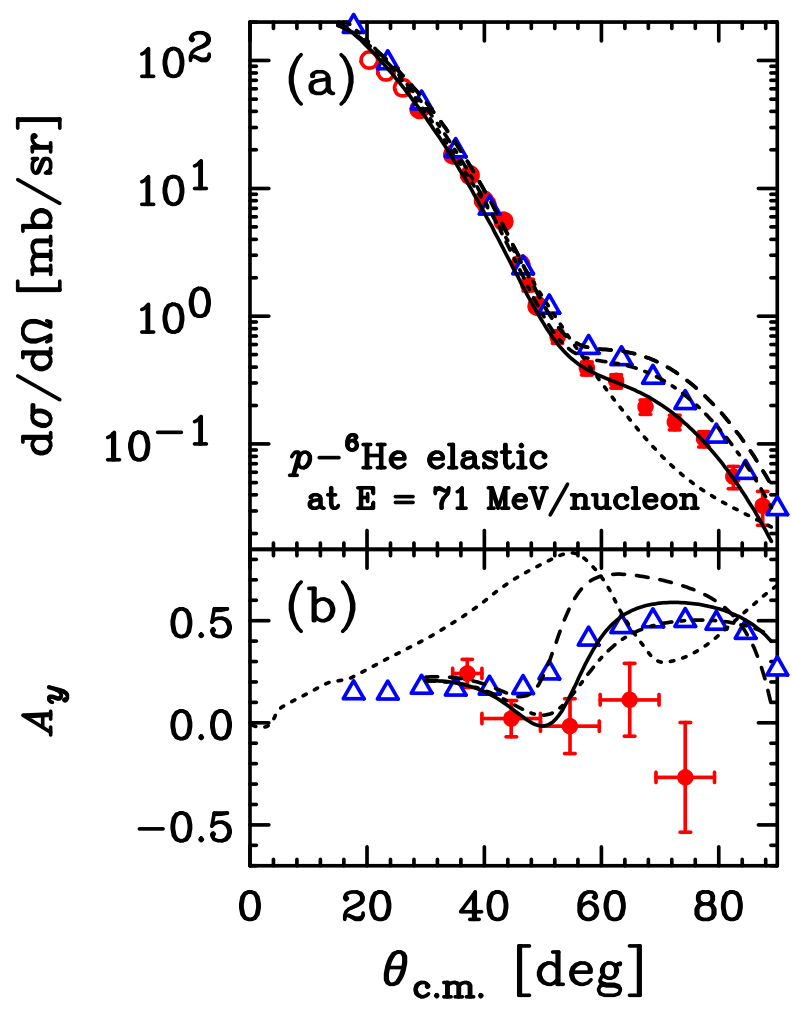

FIG. 2: (color online) Cross section and vector analyzing power for the $p-{ }^{6} \mathrm{He}$ elastic scattering at $71 \mathrm{MeV} /$ nucleon (filled circles), together with cross section data in Ref. [4] (open circles) and data for ${ }^{6} \mathrm{Li}$ [19] (triangles) targets. Dotted lines is a $t$-folding calculation in Ref. 21]. Results of $6 \mathrm{BF}$ calculations with harmonic oscillator (dashed), WS with (solid) and without halo (dot-dashed) single particle wave functions are shown.

can find small but apparent differences at backward angles $\theta_{\text {c.m. }}>50^{\circ}$, which can be a manifestation of a halo structure in $\left.{ }^{6} \mathrm{He} 20\right]$.

In the lower panel of Fig. 2, we show the analyzing power for the $p-{ }^{6} \mathrm{He}$ elastic scattering at $71 \mathrm{MeV} /$ nucleon, together with the ${ }^{6} \mathrm{Li}$ data. In sharp contrast to the cross section, the $A_{y}$ data for the $p-{ }^{6} \mathrm{He}$ scattering are quite different from that for $p-{ }^{6} \mathrm{Li}$, especially at $\theta_{\text {c.m. }}>50^{\circ}$. Dotted lines in Fig. 22represent prediction of the full $t$-folding optical potential model by Weppner et al. 21] reported before our measurement. This model calculates the $p$ $A$ scattering amplitudes by folding free nucleon-nucleon scattering amplitudes ( $t$-matrix) based on the Nijmegen I interaction with off-shell density matrices. The calculations predict large positive values of analyzing power in the region of measurement independent of the nuclear structure model used. The predicted angular distribution is clearly inconsistent with the present $A_{y}$ data, while the calculation reproduces the cross section data at forward angles reasonably. Those comparisons indicate that vector analyzing power can provide new information on the reaction mechanism and also on the nuclear structure, additional to that from the smaller scale effects in the elastic scattering cross section.

To obtain deeper understanding, we have compared the data with two different $g$-matrix folding model calculations: one is a full six-body folding $(6 \mathrm{BF})$ calculation [20, 22] and the other is a cluster-folding (CF) calculation which considers ${ }^{6} \mathrm{He}$ to have an explicit $\alpha$-core.

The $g$-matrix folding model, defined and used in Refs. [20, 22], has been successful in describing $p$ - $A$ elastic and inelastic scatterings for a wide range of nuclear masses and energies. In the model, the non-locality of the $p$ - $A$ interaction due to an exchange term is taken into account in a fully microscopic way. Nuclear structure effects to the scattering are taken into account through single particle wave functions, one-body density matrix elements, and the $g$-matrix interaction. The $g$-matrix is obtained by solving the Bethe-Bruckner-Goldstone equations for Bonn-B potential. Use of the $g$-matrix is the largest difference from that in Ref. [21]. Three curves in Fig. 2 represent results for different nuclear structure models: solid and dot-dashed curves are results with a single particle wave function for a Woods-Saxon (WS) potential with and without a halo component, respectively, while dashes curves are for a harmonic oscillator potential. It is found that the predicted $A_{y}$ varies by as much as 0.2 depending on the nuclear structure model used in the analysis. Overall agreement to the $d \sigma / d \Omega$ and $A_{y}$ data can be obtained with WS wave functions. In particular $d \sigma / d \Omega$ at $\theta_{\text {c.m. }}>50^{\circ}$ prefer a model with halo structure.

What is the origin of this sensitivity to nuclear structure? Is it due to the direct valence neutron contribution or to the $\alpha$-core contribution, or both? Comparison with CF calculations is suited for clarifying the origin. Since the ${ }^{6} \mathrm{He}$ nucleus is known to have a welldeveloped $\alpha-n-n$ structure, a folded interaction of $p-\alpha$ and $p-n$ interactions with an $\alpha-n-n$ cluster distribution should be a good approximation to the $p-{ }^{6} \mathrm{He}$ interaction potential. The $\mathrm{CF}$ optical potential can be written as $U_{\mathrm{CF}}=\sum_{i=1,2} \int V_{p n_{i}} \rho_{n}\left(r_{i}\right) d \boldsymbol{r}_{i}+\int V_{p \alpha} \rho_{\alpha}\left(r_{\alpha}\right) d \boldsymbol{r}_{\alpha}$, where $V_{p X}$ includes both central and spin-orbit parts. In the actual calculation, a phenomenological optical potential which reproduces the $p-{ }^{4} \mathrm{He}$ elastic scattering data at $72 \mathrm{MeV} /$ nucleon [23] is used as the $p$ - $\alpha$ interaction. Complicated effects in the $p$ - $\alpha$ interaction, such as non-locality due to exchange process, are considered to be simulated by the phenomenological optical potential, at least in part. The complex effective interaction (CEG) 24] is adopted as the $p-n$ interaction. Those interactions are folded with the $\alpha-n-n$ distributions determined by using Gaussian expansion method 25. Details of the calculation will be reported elsewhere 26].

In Fig. 3, results of the CF calculation (solid lines) are compared with the ${ }^{6} \mathrm{He}$ (circles) and ${ }^{4} \mathrm{He}$ (squares) 23] data. The ${ }^{4} \mathrm{He}$ data are plotted at the angle where momentum transfer for $p$ - ${ }^{6} \mathrm{He}$ is the same as that for 
the corresponding $p$ - ${ }^{4} \mathrm{He}$ data. Although the angular distribution of $d \sigma / d \Omega$ for ${ }^{6} \mathrm{He}$ differs considerably from the more gradual one for ${ }^{4} \mathrm{He}$, the $A_{y}$ data are similar to each other. The CF calculation reproduces both of $d \sigma / d \Omega$ and $A_{y}$ reasonably, in particular at $\theta_{\text {c.m. }} \sim 35^{\circ}-60^{\circ}$.

To separate the valence neutron and the $\alpha$-core contributions, calculations with $V_{p n ; \ell s}=0$ (dashed lines) and with $V_{p n ; \ell s}=V_{p n ; \text { central }}=0$ (dot-dashed lines) have been made. The latter corresponds to extraction of a "pure" $\alpha$-core contribution. As shown in Fig. 3 the $p$ - $n$ central interaction causes a sizable effect on $d \sigma / d \Omega$. Due to the $\alpha$-core motion in ${ }^{6} \mathrm{He}$, matter distribution of the core part is wider than that of a bare ${ }^{4} \mathrm{He}$ nucleus, while it is naturally narrower than that of ${ }^{6} \mathrm{He}$ as a whole. Reflecting this, $d \sigma / d \Omega$ for the $\alpha$-core contribution appears between ${ }^{4} \mathrm{He}$ and ${ }^{6} \mathrm{He}$ data. It is also found that the spin-orbit interaction $V_{p n ; \ell s}$ gives negligible effects on $d \sigma / d \Omega$ and $A_{y}$, which is consistent with predictions in Ref. [16]. Thus, from comparisons with $\mathrm{CF}$, it is concluded that nuclear structure sensitivity of $A_{y}$ does not originate from the direct valence neutron contribution, but from the $\alpha$-core contribution. The latter, which is affected by recoil of the valence neutrons, seems to be a possible key to understand the behavior of $A_{y}$.

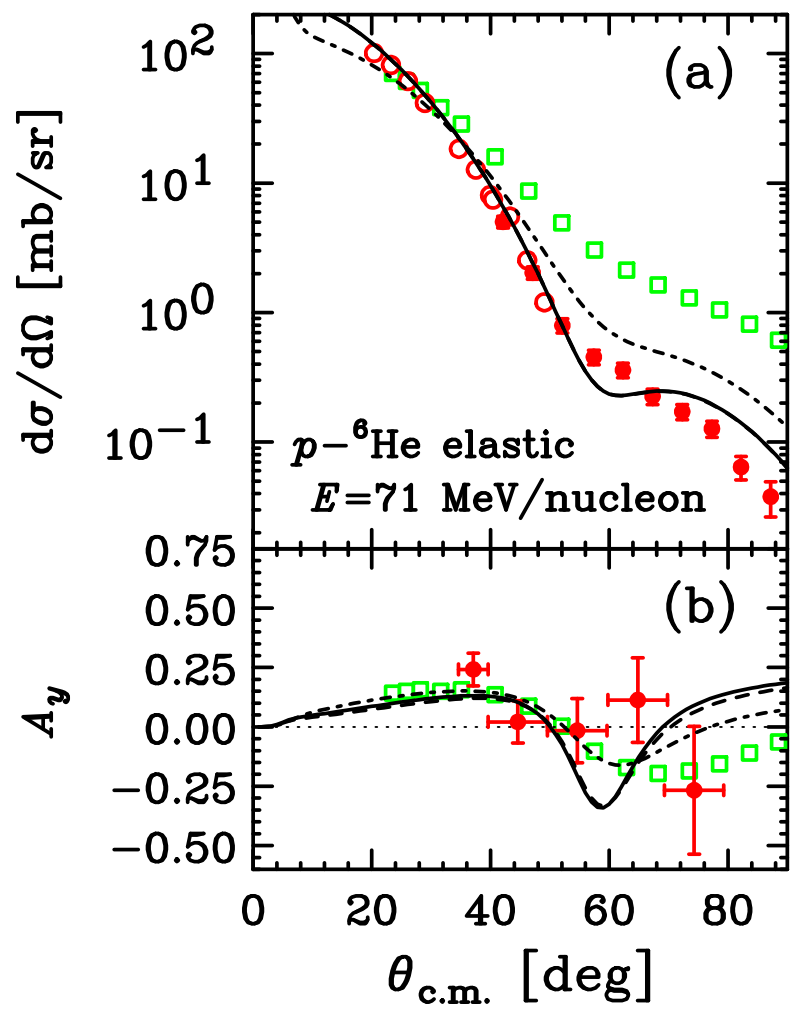

FIG. 3: (color online) Present data compared with the clusterfolding calculations. Solid, dashed, and dot-dashed lines represent calculations with full, $V_{p n ; \ell s}=0$, and $V_{p n ; \ell s}=$ $V_{p n ; \text { central }}=0$ interactions, respectively. Data for the $p-{ }^{4} \mathrm{He}$ scattering 23] (squares) are also shown.
In summary, we have performed an experiment to measure $A_{y}$ and $d \sigma / d \Omega$ in scattering of a neutron-rich ${ }^{6} \mathrm{He}$ nucleus from protons, by using the newly-developed polarized proton solid target. The $A_{y}$ data are obtained with a proton polarization determined by asymmetry in the $p$ - ${ }^{4} \mathrm{He}$ scattering. We conclude, from comparisons with the microscopic folding model calculations, that (1) overall agreement between the present data and $6 \mathrm{BF}$ calculation is found for WS wave function. In particular, the $d \sigma / d \Omega$ data at backward angles favor existence of halo structure in ${ }^{6} \mathrm{He}$; (2) the data are reproduced by $\mathrm{CF}$ calculations reasonably well. The CF calculations show that direct contribution from the valence neutrons to analyzing power is negligibly small. Thus nuclear structure effects on $A_{y}$ may originate from the spatial distribution of the $\alpha$-core in ${ }^{6} \mathrm{He}$, which is closely connected to the valence neutron distribution. This can be a possible key to understand spin-orbit coupling in a neutron-rich ${ }^{6} \mathrm{He}$ nucleus.

The present work has demonstrated that the technique to polarize protons in a low magnetic field can open a new possibility to explore physics of unstable nuclei. Experiments with the target would provide fruitful results in future radioactive nuclear beam facilities.

The authors thank the RIKEN and CNS staffs for operation of accelerators during the measurement. One of the authors (S.S.) expresses his gratitude for financial support by a Grant-in-Aid for Japan Society for the Promotion of Science (JSPS) Fellows (No. 18-11398). This work was supported by the Grant-in-Aid No. 17684005 of the Ministry of Education, Culture, Sports, Science, and Technology of Japan.

* Electronic address: uesaka@cns.s.u-tokyo.ac.jp

[1] C. Oxley et al., Phys. Rev. 91, 419 (1953).

[2] O. Chamberlain et al., Phys. Rev. 102, 1659 (1956).

[3] E. Fermi, Nuovo Cimento 10, 407 (1954).

[4] A. Korsheninnikov et al., Nucl. Phys. A 617, 45 (1997).

[5] R. Wolski et al., Phys. Lett. B 467, 8 (1999).

[6] M. D. Cortina-Gil et al., Phys. Lett. B 401, 9 (1997).

[7] A. Lagoyannis et al., Phys. Lett. B 518, 27 (2001).

[8] P. Egelhof et al., Eur. Phys. Jour. A 15, 27 (2002).

[9] G. D. Alkhazov et al., Nucl. Phys. A 712, 269 (2002).

[10] S. Goertz, W. Meyer, and G. Reicherz, Prog. Part. Nucl. Phys. 49, 403 (2002), and references therein.

[11] T. Wakui, in Proc. XIth Int. Workshop on Polarized Ion Source and Polarized Gas Targets 2005, edited by T. Uesaka, H. Sakai, A. Yoshimi, and K. Asahi (World Scientific, Singapore, 2007), p. 49.

[12] A. Henstra, P. Dirksen, and W. T. Wenckebach, Phys. Lett. A 134, 134 (1988).

[13] T. Kubo et al., Nucl. Instr. Meth. B 70, 309 (1992).

[14] T. Wakui et al., Nucl. Instr. Meth. A 550, 521 (2005).

[15] M. Hatano et al., Eur. Phys. Jour. A 25, 255 (2005).

[16] R. Crespo and A. M. Moro, Phys. Rev. C 76, 054607 (2007). 
[17] H. Togawa and H. Sakaguchi, RCNP Annual Report 1 (1987).

[18] T. Uesaka et al., Nucl. Instr. and Meth. A 526, 186 (2004).

[19] R. Henneck et al., Nucl. Phys. A 571, 541 (1994).

[20] S. V. Stepantsov et al., Phys. Letts. B542, 35 (2002).

[21] S. P. Weppner, O. Garcia, and Ch. Elster, Phys. Rev. C 61, 044601 (2000).
[22] K. Amos et al., Adv. Nucl. Phys. 25, 275 (2001).

[23] S. Burzynski et al., Phys. Rev. C 39, 56 (1989).

[24] N. Yamaguchi, S. Nagata, and T. Matsuda, Prog. Theor. Phys. 70, 459 (1983).

[25] E. Hiyama, Y. Kino, and M. Kamimura, Prog. Part. Nucl. Phys. 51, 223 (2003).

[26] S. Sakaguchi, Y. Iseri et al., in preparation. 